\title{
An Unusual Case of Chest Pain in a Young Adult
}

\author{
${ }^{1}$ Nabeel A Hashmi, ${ }^{2}$ Sourya Acharya, ${ }^{3}$ Shashiraj Lahoti, ${ }^{4}$ Saish Alegaonkar, ${ }^{5}$ Samarth Shukla
}

\begin{abstract}
Echinococcosis is an infection caused in humans by Echinococcus granulosus which produce cystic hydatid disease. It is prevalent in most areas with livestock is raised in association with dogs. Liver is the most common site of this infection. We are presenting a case of 19-year-old adult who had chronic cough and pleuritic pain due to hydatid cyst in left lung.
\end{abstract}

Keywords: Echinococcosis, Hydatid cyst, Liver, Lung.

How to cite this article: Hashmi NA, Acharya S, Lahoti S Alegaonkar S, Shukla S. An Unusual Case of Chest Pain in a Young Adult. Int J Recent Surg Med Sci 2017;3(1):56-57.

\section{Source of support: Nil}

Conflict of interest: None

\section{INTRODUCTION}

Hydatid disease is a parasitic infection characterized by formation of cysts mostly in liver and lungs. It is caused by larval stage of Echinococcus granulosus. Other species include Echinococcus vogeli and Echinococcus multilocularis. Echinococcus species have definite host which are canines and intermediate host in cattle, goats, humans. It is prevalent in China, Central Asia, Middle East, Eastern Africa. Hydatid disease has no specific signs and symptoms. This disease presents with constitutional symptoms, such as fever, cough with expectoration. ${ }^{1}$

\section{CASE REPORT}

A 19-year-old male presented to medicine outpatient department (OPD) with chief complaints of chest pain on left side since 1 month, and cough since 7 days. The pain was sharp and localized in the left mammary area, which was aggravated by deep inspiration and coughing. There was no history of fever, hemoptysis, expectoration, palpitations, dyspnea, paroxysmal nocturnal dyspnea, and orthopnea. He was laborer by occupation.

\footnotetext{
${ }^{1,3,4}$ Resident, ${ }^{2,5}$ Professor

${ }^{1-4}$ Department of Medicine, Datta Meghe Institute of Medical Sciences, Wardha, Maharashtra, India

${ }^{5}$ Department of Pathology, Datta Meghe Institute of Medical Sciences, Wardha, Maharashtra, India

Corresponding Author: Nabeel A Hashmi, Resident Department of Medicine, Datta Meghe Institute of Medical Sciences, Wardha, Maharashtra, India, Phone: +919689323925 e-mail: nabhas88@yahoo.in
}

On general examination, the patient was tachycardic, respiratory rate was $22 / \mathrm{min}$, blood pressure $120 / 70 \mathrm{~mm} \mathrm{Hg}$. There was no clubbing, cyanosis, raised jugular venous pressure, or edema feet.

Respiratory system examination revealed diminished chest movement in left mammary, inframammary, left intraaxillary, and posteriorly left infrascapular area. On auscultation air entry was diminished and bronchial breathing was heard anteriorly in left inframammary area.

Other systemic examinations were normal. Complete blood count $(\mathrm{CBC})$, total cholesterol, ESR were within normal limits. Chest X-ray (CXR) revealed homogeneous rounded opacity in the left mid-zone with surrounding consolidation (Fig. 1).

High resolution computed tomography (CT) thorax revealed a large well-defined nonenhancing cystic mass of size $8.3 \times 6.7 \times 5.3 \mathrm{~cm}$ with thin irregular walls in the lingular segment of left upper lobe. The lesion shows undulating walls with perilesional consolidation and multiple centrilobular nodules with interlobular septal thickening, suggestive of hydatid cyst in left upper lobe (Fig. 2). On asking leading questions, the patient denied any contact with dogs. This may be explained by the fact that eggs are partially resistant to drying and can remain viable for many weeks, and food (raw vegetables) can become contaminated without coming in direct contact with infected animals.

Patient was started on tablet albendazole $15 \mathrm{mg} / \mathrm{kg} /$ day, and was referred to surgery. Percutaneous aspiration,

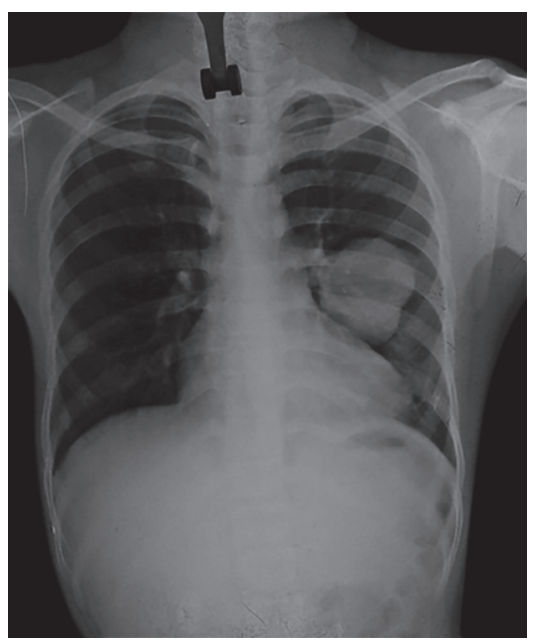

Fig. 1: Chest X-ray showing homogenous opacity in left midzone with regular margins with surrounding consolidation 

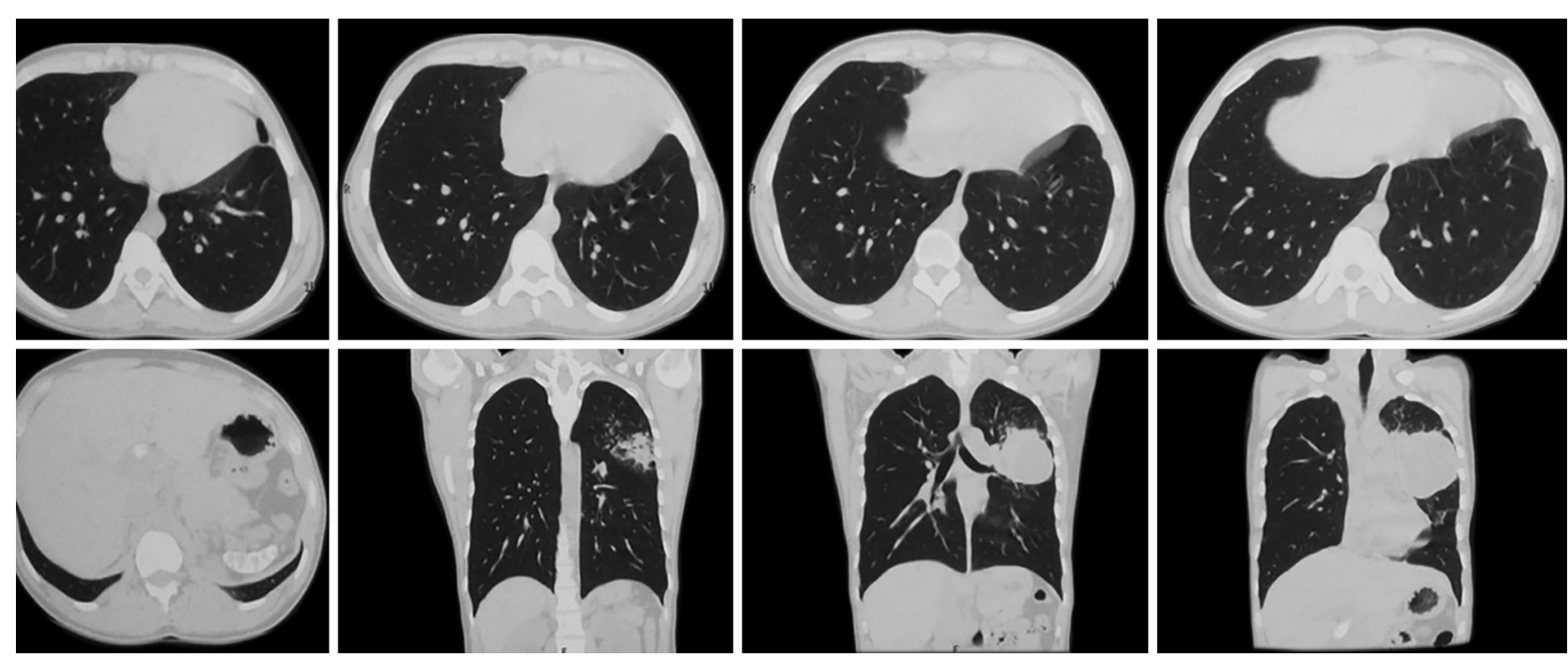

Fig. 2: Hydatid cyst

infusion of scolicidal agents, and reaspiration (PAIR) was done and the patient was discharged on tablet albendazole $400 \mathrm{mg}$ bid for 28 days and is awaiting follow-up.

\section{DISCUSSION}

Hydatid disease is a parasitic infection caused by E. granulosus. It is endemic in many countries. It occurs due to ingestion of hexacanth egg of dog tapeworm. Generally, there are no specific signs and symptoms, and some patients may even be asymptomatic. Symptoms depend on site and size of lesions. Liver and lungs are the most common sites. ${ }^{2}$

Nonproductive cough and minimal hemoptysis are most prevalent symptoms of hydatid cyst of lung. Our case presented with chest pain, which was pleuritic in nature. The cough in our case was predominantly dry, which was, may be, due to consolidation with pleuritis. When the cyst ruptures, there is abrupt onset of cough, expectoration, fever, or acute hypersensitivity reaction that mimics anaphylactic shock. The sputum may become purulent or may contain fragments of hydatid membrane. Other symptoms, such as Horner's Syndrome from superior sulcus cysts, bone pain from posterior mediastinal cysts, dyspnea from tracheal compression, hemorrhage due to erosion of great vessels, and tension pneumothorax may occur depending on the site.

Work ups for hydatid cyst include CBC, ESR, CXR, ultrasonography thorax, CT thorax, and sputum analysis. Different serological tests, like enzyme-linked immunosorbent assay, latex agglutination tests, are also helpful.
Medical treatment results in reduction of cyst size. Tablet albendazole $400 \mathrm{mg}$ twice a day for 28 days and repeated from 1 to 8 times, separated by drug-free interval of 2 to 3 weeks is effective for liver and lung cysts. Adjunctive chemotherapy before and after surgery reduces the risk of recurrence by inactivating protoscolices and reduces the tension of cysts for easy removal. For uncomplicated lesion PAIR is now indicated. It is contraindicated for superficially located cyst and for cyst with multiple thick internal divisions. Principles of mandatory surgical intervention are evacuation of the cyst with removal of endocyst, avoidance of contamination, and management of residual cavity. ${ }^{3-5}$

\section{REFERENCES}

1. White, C.A. Jr.; Weller, P.F. Cestode infections. In: Kasper, D.L.; Fauci, A.S.; Hauser, S.L.; Longo, D.L.; Jamison, J.L.; Loscalzo, J., editors. Harrison's principles of internal medicine. 19th ed., Vol. 2. New York, NY: McGraw Hill Education; 2015. p. 1432-1434.

2. Ghallab NH, Alsabahi AA. Giant viable hydatid cyst of the lung: a case report. J Med Case Reports 2:359-363.

3. Karaoglanoglu N, Kurkcuoglu IC, Gorguner M, Eroglu A, Turkylimaz A. Giant hydatid lung cysts. Eur J Cardiothorac Surg 2001;19(6):914-917.

4. Ellaban A, Elzayat S, Elmuzaien M, Nasher A, Homesh N, Alabsi M. The effect of preoperative albendazole in the treatment of liver hydatid cysts. Egyptian J Med Lab Sci 1994;15:309-319.

5. Ayles HM, Corbett EL, Taylor I, Cowie AGG, Bligh J, Walmsley K, Bryceson ADM. A combined medical and surgical approach to hydatid disease: 12 years experience at the Hospital for Tropical Disease, London. Ann R Coll Surg Engl 2002 Mar;84(2):100-105. 